\title{
Numerical modeling of the disappearance of equatorial plasma bubble by nighttime medium- scale traveling ionospheric disturbances
}

\section{Min-Yang Chou ( $\square$ chou@ucar.edu )}

University Corporation for Atmospheric Research https://orcid.org/0000-0001-7172-9569

Charles C. H. Lin

National Cheng Kung University Department of Earth Sciences

Joseph D. Huba

Syntek Technologies

Full paper

Keywords: Equatorial Plasma Bubble, Nighttime medium-scale traveling ionospheric disturbances, Electrodynamical coupling, Numerical simulation

Posted Date: January 26th, 2021

DOI: https://doi.org/10.21203/rs.3.rs-102487/v2

License: (c) (i) This work is licensed under a Creative Commons Attribution 4.0 International License. Read Full License 
1 Numerical modeling of the disappearance of equatorial plasma bubble by

2 nighttime medium-scale traveling ionospheric disturbances

3 Author \#1: Min-Yang Chou, COSMIC Program Office, University Corporation for

5 Author \#2: Charles C. H. Lin, Department of Earth Sciences, National Cheng Kung University,

7 Author \#3: Joseph D. Huba, Syntek Technologies, Fairfax, VA, USA, jdhuba@gmail.com

8 corresponding author

9 Min-Yang Chou 


\section{Abstract}

11 The Naval Research Laboratory first-principles ionosphere model SAMI3/ESF is performed to

12 study the interaction between the nighttime medium-scale traveling ionospheric disturbances

13 (MSTIDs) and equatorial plasma bubbles (EPBs). The synthetic dynamo currents are imposed

14 into the potential equation to induce polarization electric fields for generating the MSTIDs.

15 Simulations demonstrate that the MSTIDs can inhibit the upward growth of EPBs; however,

16 MSTIDs alone are insufficient to explain the disappearance of EPBs. We found that the

17 meridional winds likely contribute to the disappearance of MSTIDs by reducing the background

18 electron density and polarization electric fields within the EPBs. Then, the MSTIDs transport

19 plasma to fill the EPB depletions up via $\mathbf{E} \times \mathbf{B}$ drifts. Both MSTIDs and meridional winds are

20 necessary to comprehend the underlying mechanism of EPB disappearance. We also found that

21 the zonal and vertical $\mathbf{E} \times \mathbf{B}$ drifts within the MSTIDs affect the morphology of EPBs, leading

22 to a reverse-C shape structure.

23

\section{Keywords}

25 1. Equatorial Plasma Bubble 
26 2. Nighttime medium-scale traveling ionospheric disturbances

27 3. Electrodynamical coupling

28 4. Numerical simulation

\section{Main Text}

\section{1. Introduction}

medium-scale traveling ionospheric disturbances (MSTIDs) are often observed in the nighttime equatorial and mid-latitude ionosphere. They are electrodynamical structures generated by the generalized Rayleigh-Taylor instability (e.g., Kelley, 1989) and Perkins instability (Perkins, 1978), respectively. Dungey (1956) first proposed the Rayleigh-Taylor instability as the mechanism responsible for driving EPBs. The low-density plasma moves upward into the high-density plasma region via $\mathbf{E} \times \mathbf{B}$ drifts driven by gravitational force-induced ion currents, creating ionospheric plasma depletions along magnetic flux tubes. The meridionally-elongated wedges of 
have been proposed to explain the generation of EPBs, such as the neutral winds, gravity waves,

43 and electric and magnetic fields (Sultan, 1996; Rama Rao et al., 1997; Abdu et al., 2009). The

44 pre-reversal enhancement (PRE) is considered to play a dominant role in causing the post-sunset

45 rise of the ionospheric layer, resulting in the steep vertical gradient of electron density favorable

46 for the development of EPBs (e.g., Rajesh et al., 2017).

47 On the other hand, the nighttime MSTIDs have been investigated for many years (e.g.,

48 Behnke et al. 1979; Miller et al. 1997; Saito et al. 1998; Shiokawa et al. 2003a; Rajesh et al. 2016;

49 Chou et al. 2017). Space- and ground-based observations have shown the oscillation of

50 polarization electric fields within the MSTIDs (Saito et al. 1995; Kelley et al. 2000; Shiokawa et

51 al. 2003b), supporting the hypothesis that the Perkins instability plays a crucial role in the

52 generation of MSTIDs. The polarization electric fields can further map along the geomagnetic

53 field lines, displaying conjugate structures in both hemispheres (Otsuka et al. 2004). Since the

54 theoretical growth rate of the Perkins instability is quite small, gravity wave seeding (Kelley and

55 Fukao, 1991; Huang et al. 1994; Miller et al. 1997; Li et al. 2016; Chou et al. 2017; 2018) or

56 coupling between the $\mathrm{F}$ and sporadic-E layers (EsL) (Cosgrove and Tsunoda, 2004; Tsunoda,

57 2004; Yokoyama et al. 2009) are suggested to be the plausible mechanisms to accelerate the 
growth of nighttime MSTIDs. disappearance of the EPBs by transporting the ambient plasma into the plasma-depleted region across the geomagnetic field lines via $\mathbf{E} \times \mathbf{B}$ drifts. However, the EPBs usually cause significant plasma depletions over 10 TECu (e.g., Nishioka et al. 2008) while the amplitudes of MSTIDs are within the ranges of $\sim 0.2-1 \mathrm{TECu}$ (e.g., Tsugawa et al. 2007; Chou et al. 2017), making it difficult to fade out the EPBs by filling the plasma depletions up with ambient plasma along the flux tubes.

70 Additionally, plasma diffusion across the geomagnetic field line is relatively slow compared to the field-aligned diffusion and may take a longer time to fill the depletion up. 
74 model (Huba et al. 2008) to investigate the coupling between the nighttime MSTIDs and EPBs.

75 We found that the $\mathbf{E} \times \mathbf{B}$ drifts within MSTIDs can suppress the upward motion of EPBs and

76 affect the morphology of EPBs. However, the meridional winds also play an essential role in the

77 disappearance of EPBs. Concerning the observations reported by Otsuka et al. (2012) and

78 Shiokawa et al. (2015), both MSTIDs and transequatorial winds appear to be necessary conditions

79 to explain the disappearance of EPBs.

80

81 2. The SAMI3/ESF Model

82

The NRL self-consistent SAMI3/ESF model based on the 2-D SAMI2 (Sami2 is Another

83 Model of the Ionosphere) and 3-D SAMI3 (Huba et al. 2000; Huba and Joyce, 2010) has applied

84 to study the evolution and generation of ESF (Huba et al. 2008, 2009a; Krall et al. 2013; Wu et

85 al. 2015) and nighttime MSTIDs (Duly et al. 2014; Chou et al. 2018). In this study, the SAMI2

86 model is run for $43 \mathrm{~h}$ to initial the SAMI3/ESF model. The geophysical indices used are F10.7 =

87105 (s.f.u), F10.7A = 105 (s.f.u), Ap = 37, and day-of-year $=70$. The SAMI3/ESF model uses a

88 grid $(\mathrm{nz}, \mathrm{nf}, \mathrm{nl})=(101,200,128)$ where $\mathrm{nz}$ is the number of grid points along each geomagnetic

89 field line (s direction), $\mathrm{nf}$ is the number of geomagnetic field lines in altitude ( $\mathrm{p}$ direction), and $\mathrm{nl}$ 
90 is the number in longitude ( $\phi$ direction). Since the 3D model uses an aligned diploe magnetic

91 field with a magnetic apex height from $85 \mathrm{~km}$ to $2400 \mathrm{~km}$, the geomagnetic latitude and

92 geographic latitude are the same, and the longitudinal and latitudinal width are limited to $4^{\circ}$ and

$93 \pm 30^{\circ}$ in both hemispheres. The simulations are run for $7 \mathrm{~h}$ from $\sim 19: 09 \mathrm{LT}$ to $\sim 2: 07 \mathrm{LT}$.

94 The SAMI3/ESF potential equation is solved based on current conservation $\nabla \cdot J=0$ and

95 equipotential field lines (Krall et al. 2009a; Kuo et al. 2011; Huba et al. 2015). The electric current

$96 \mathrm{~J}$ can be written as $\mathrm{J}=\mathrm{J}_{\mathrm{E}}+\mathrm{J}_{\mathrm{g}}+\mathrm{J}_{\mathrm{v}}$, where $\mathrm{J}_{\mathrm{E}}, \mathrm{J}_{\mathrm{g}}$, and $\mathrm{J}_{\mathrm{v}}$ represent current terms driven by the electric

97 field, gravity, and neutral wind. In this study, the external MSTID current terms (JMSTID) are

98 included in the potential equation to act as the EsL-F coupling for generating MSTIDs (e.g.,

99 Yokoyama et al. 2009). The modified integrated potential equation used in this study is

$100 \quad \frac{\partial}{\partial p} p \Sigma_{p p} \frac{\partial \Phi}{\partial p}-\frac{\partial}{\partial p} \Sigma_{H} \frac{\partial \Phi}{\partial \phi}+\frac{\partial}{\partial \phi} \frac{1}{p} \Sigma_{p \phi} \frac{\partial \Phi}{\partial \phi}+\frac{\partial}{\partial \phi} \Sigma_{H} \frac{\partial \Phi}{\partial p}$

$101=\frac{\partial F_{\phi g}}{\partial \phi}+\frac{\partial F_{\phi V}}{\partial \phi}-\frac{\partial F_{p g}}{\partial p}+\frac{\partial F_{p V}}{\partial p}+\frac{\partial F_{p E}}{\partial p}+\frac{\partial F_{\phi E}}{\partial \phi}$

102 where $F_{\phi g}=\int\left(r_{E} \sin ^{3} \theta / \Delta\right)\left(B_{0} / c\right) \sigma_{H c} g_{p} d s, F_{\phi V}=\int\left(r_{E} \sin ^{3} \theta / \Delta\right)\left(B_{0} / c\right)\left(\sigma_{H} V_{n \phi}-\sigma_{P} V_{n p}\right) d s$,

$103 F_{p g}=-\int r \sin \theta\left(B_{0} / c\right) \sigma_{P c} g_{p} d s$, and $F_{p V}=\int r \sin \theta\left(B_{0} / c\right)\left(\sigma_{P} V_{n \phi}+\sigma_{H} V_{n p}\right) d s, \Phi$ is the electrostatic

104 potential, $g_{p}$ is the component of gravity perpendicular to $\mathbf{B}, V_{n}$ is perpendicular wind component.

105 The detailed definitions of the variables are given by Huba and Joyce (2010). The MSTID current 
106 terms are $F_{\phi E}=\int r^{3} / \mathrm{r}_{E}^{3}\left(\sin ^{3} \theta / \Delta^{2}\right)\left(\sigma_{P} E_{\phi}+\sigma_{H} E_{p}\right) d s$ and $F_{p E}=\int r \sin \theta / \Delta\left(\mathrm{r} / \mathrm{r}_{\mathrm{E}}\right)^{3}\left(\sigma_{p} E_{\mathrm{p}}-\sigma_{H} E_{\phi}\right) d s$. The $E_{p}$

107 and $E_{\phi}$ are defined as

$108 E_{[\mathrm{p}, \phi]}=-A_{E} \frac{1}{1+e^{-\left(\text {lat }- \text { lat }_{0}\right)}} e^{-\frac{\left(\text { lon-lon }_{0}\right)^{2}}{2 w^{2}}} \frac{k_{[x, y]}}{k} \sin \left(k_{x} x+k_{y} y-\omega t\right)$

109 where $A_{E}$ is the amplitude of electric field, $k_{x}=k \cos \theta_{T I D}, k_{y}=k \sin \theta_{T I D}, \omega=\frac{2 \pi}{T}, \mathrm{t}$ is

110 time, $\mathrm{k}$ is the wavenumber, lon and lat are longitude and latitude, and $\theta_{T I D}$ is the angle between

111 the direction normal to the frontal structure of MSTIDs and the geomagnetic east. We assume

$112 k=\frac{2 \pi}{2^{\circ}}, \theta_{T I D}=30^{\circ}$ and $\mathrm{T}=70 \mathrm{~min}$, which are similar to the MSTIDs observations (e.g.,

113 Shiokawa et al. 2003b). The $A_{E}$ is set to $2 \mathrm{mV} / \mathrm{m}$ (cf. Pfaff et al. 2005). The smooth function

$114 \frac{1}{1+e^{-\left(l a t-l a t_{0}\right)}} e^{-\frac{(\text { lon-lon })^{2}}{2 \times w^{2}}}$ is used to limit the latitudinal and longitudinal extension of current

115 oscillations. w, lat 0 , and $\operatorname{lon}_{0}$ are set to $1.25^{\circ}, 17^{\circ}$ and $2^{\circ}$, respectively, so that the currents will

116 gradually decrease to zero below $17^{\circ} \mathrm{N}$ at $85 \mathrm{~km}$ and the boundary effect will be reduced. The

117 MSTID current terms are confined to the altitudes of $85-450 \mathrm{~km}$ in the northern hemisphere and

118 will self-consistently generate polarization electric fields $(E=-\nabla \Phi)$ along the field lines to satisfy

$119 \nabla \cdot J=0$ and equipotential field line assumption, leading to a conjugate effect (e.g., Saito et al.,

120 1995; Otsuka et al. 2004).

121 Similar methods were used by Huba et al. (2015) and Kuo et al. (2011). Huba et al. (2015) 
122 simulated the tsunami-induced gravity waves by imposing neutral wind perturbations (U') in the

123 ionospheric F region. The dynamo currents driven by wind perturbations self-consistently induce

124 polarization electric fields mapping to the conjugate region. Kuo et al. (2011) studied the

125 lithosphere-atmosphere-ionosphere coupling by imposed an external dynamo current from the

126 atmosphere at $85 \mathrm{~km}$ altitude (the lower boundary for the SAMI3 ionosphere). The dynamo

127 current flowing into the ionosphere causes significant ionospheric perturbations in both

128 hemispheres and leads to the EPB formation.

129 The EPBs are simulated by imposing a Gaussian perturbation in the ion density with an

130 amplitude $\mathrm{A}_{\mathrm{m}}$ of $10 \%$ in the bottomside $\mathrm{F}$ layer.

$131 \quad N^{\prime}=N_{0}\left(1-A_{m} e^{-\frac{\left(l o n-l o n_{0}\right)^{2}}{2 w^{2}}}\right)$

132 where $N_{0}$ and $N^{\prime}$ are the initial and perturbed ion densities. The perturbation is centered at lono

$133=2^{\circ}$, and $w=0.3^{\circ}$. The gravity-driven current terms $\left(F_{p g}, F_{\phi g}\right)$ of equation (1) are the primary

134 source terms to drive the growth of EPBs.

\section{3. Results}

137 Three SAMI3/ESF numerical simulations are performed using zero neutral wind conditions. 
138 Case 1 simulates the generation and evolution of EPB; case 2 and case 3 simulate the impacts of

139 MSTIDs on the EPBs in the onset and structure (or mature) phases by imposing synthetic MSTIDs

140 at 22:00 LT and 23:30 LT, respectively. The onset phase demonstrates that the EPBs develop from

141 the crest of upwellings and continuously grow upward and poleward; the structure phase

142 demonstrates that the EPBs are well-developed and will become fossil (e.g., Tsunoda et al. 2015).

143 Figure 1 shows the time sequence of electron density contours and vertical $\mathbf{E} \times \mathbf{B}$ drifts in

144 contour lines (black and white lines) as a function of longitude and altitude on a magnetic

145 equatorial plane for cases 1 (left column), 2 (middle column), and 3 (right column), respectively

146 (see also additional files 1-3 in supporting information). The black and white contour lines

147 indicate the downward and upward $\mathbf{E} \times \mathbf{B}$ drifts, respectively. The yellow and red stars indicate

148 the positions of peak upward $\mathbf{E} \times \mathbf{B}$ drifts of the EPB and topside EPB $(>900 \mathrm{~km})$. Dense white

149 contour lines within the plume structures are visible, demonstrating strong upward $\mathbf{E} \times \mathbf{B}$ drifts

150 within the EPB. The EPB starts to grow from the bottomside ionosphere after $\sim 22: 00$ LT. Case 1

151 shows that the EPB intrudes into $\sim 1200 \mathrm{~km}$ altitude at 01:49 LT, displaying a distinct plume 
154 EPB in the onset phase has a peak upward $\mathbf{E} \times \mathbf{B}$ drift of $\sim 686 \mathrm{~m} / \mathrm{s}$ in the $\mathrm{F}$ region at 22:47 LT

155 and eventually decrease to $364 \mathrm{~m} / \mathrm{s}$ in the structure phase at 01:49 LT. The topside EPB above

$156900 \mathrm{~km}$ has weaker peak vertical drifts ranging from 136 to $156 \mathrm{~m} / \mathrm{s}$.

157 It is noteworthy that EPBs usually occur during 19:00-20:00 LT because the PRE can

158 accelerate the growth rate. The simulated EPBs develop after 22:00 LT are due to the background

159 conditions (e.g., Sultan, 1996). SAMI3/ESF uses the empirical thermosphere models

160 NRLMSIS00 (Picone et al., 2002) to specify the neutral species. The background conditions may

161 be different from the real situation, which in turn affect the growth rate (e.g., Huba and Liu, 2020).

162 However, EPBs could occur after 22:00 LT in June solstice (Yizengaw et al., 2013), making the

163 MSTIDs that often occur after 21:00 LT around solstice to have a higher probability of

164 encountering the EPBs. Nevertheless, the onset time of EPBs will not affect the electrodynamics

165 between the EPBs and MSTIDs.

166 In case 2, ionospheric undulations due to the MSTIDs are discernible in the topside

167 ionosphere. The MSTIDs have vertical $\mathbf{E} \times \mathbf{B}$ drifts of $\sim \pm 10-50 \mathrm{~m} / \mathrm{s}$, which agrees reasonably

168 well with the MSTIDs observations (e.g., Shiokawa et al., 2003b). While the MSTIDs encounter

169 the EPB in the onset phase, the MSTIDs suppress the EPB growth and eventually confine the 
170 EPB within $\sim 1100 \mathrm{~km}$ altitude at 01:49 LT. The peak upward $\mathbf{E} \times \mathbf{B}$ drifts of the topside EPB

171 decrease to $\sim 122 \mathrm{~m} / \mathrm{s}$ while encountering the downward $\mathbf{E} \times \mathbf{B}$ drifts of MSTIDs at 23:48 LT.

172 Bifurcations on the east wall of the plume are suppressed as well. However, the upward $\mathbf{E} \times \mathbf{B}$

173 drifts of MSTIDs also favor the growth of EPB at 00:48 and 01:49 LT, leading to the increase of

174 peak upward $\mathbf{E} \times \mathbf{B}$ drifts within the topside EPB. Of particular interest is that the MSTIDs affect

175 the morphology of EPB. The EPB stretches along with the band structure of MSTIDs, displaying

176 a reverse $\mathrm{C}$ shape of EPB. In case 3, the MSTIDs also distort and suppress the EPB in the structure

177 phase. The primary plume is confined within $\sim 1100 \mathrm{~km}$ altitude, and the upward $\mathbf{E} \times \mathbf{B}$ drifts of

178 topside EPB decrease to $\sim 91 \mathrm{~m} / \mathrm{s}$ at 01:49 LT. In general, the peak upward $\mathbf{E} \times \mathbf{B}$ drifts of the

179 bottomside EPBs are similar for three cases; however, the MSTIDs significantly impact the

180 morphology of EPBs above the F region in the onset and structure phases.

181 We further calculate the OI 630-nm airglow emission rate based on Sobral et al. (1993) to

182 compare with the results of Otsuka et al. (2012). Figure 2 shows the time sequence of the airglow

183 intensity deviation maps at $250 \mathrm{~km}$ altitude as a function of longitude and latitude for cases 1 (left

184 column), 2 (middle column), and 3 (right column). Additional movie files show this in more detail

185 [see Additional files 4-6]. The airglow intensity deviation $\left(I_{p}\right)$ is defined as $I_{p}=\left(I-I_{c}\right) / I_{c}$, where $I_{c}$ is 
186 the control run without the EPBs, and I is the OI 630-nm airglow emission rate for cases 1-3. Note

187 that the background airglow intensity deviation can reach over $20 \%$; we limit the color ranges

188 between $-1-2 \%$ to highlight the EPB variations. Case 1 shows the evolution of EPB with airglow

189 depletion. The EPB extends to about $21^{\circ} \mathrm{N}$ at $01: 49$ LT. MSTIDs can be identified with airglow

190 enhancements and depletions above $13^{\circ} \mathrm{N}$ in cases 2 and 3. Cases 2 and 3 show that MSTIDs

191 affect the latitudinal extent and morphology of EPBs, confining the EPBs within $21^{\circ} \mathrm{N}$. However,

192 the EPBs are still distinct, implying that MSTIDs alone are insufficient to explain the

193 disappearance of EPBs.

194 Otsuka et al. (2012) showed an increase of OI 630-nm airglow intensity while the EPB

195 encountered the MSTIDs. Shiokawa et al. (2015) also showed clearly airglow intensity decrease

196 at Darwin, Australia, and a slight increase at Sata, Japan, while the EPBs encountered the TIDs

197 at geomagnetically conjugate points. The sudden F-layer rise (descent) can cause a decrease

198 (increase) of the 630-nm airglow intensity because the 630-nm airglow intensity is proportional

199 to the $\mathrm{O}^{+}$and $\mathrm{O}_{2}$ density, and rising (descending) F-layer will encounter decreasing (increasing)

200 ambient $\mathrm{O}_{2}$ density. The airglow intensity enhancement implies that the transequatorial wind may

201 contribute to the disappearance of EPBs. 
on the EPBs in the structure phase and incorporate the southward winds $V=-60 \tanh (0.02 \times$

204 (altitude -100)) $\mathrm{m} / \mathrm{s}$ into case 1 and case 3 at 23:30 LT, referred to as case 4 and case 5. The

205 southward winds can reach a peak of $60 \mathrm{~m} / \mathrm{s}$ above $250 \mathrm{~km}$ and are assumed to be $0 \mathrm{~m} / \mathrm{s}$ under

$206 \quad 100 \mathrm{~km}$.

207 Figure 3 is the same as Figure 2 but for case 4 (top panel) and case 5 (bottom panel) in both

208 hemispheres. Additional movie files show this in more detail [see Additional files 7-8].

209 Transparent meridionally-elongated wedges of airglow depletions related to EPBs are visible in

210 both cases. Significant airglow enhancements on either side of EPBs are visible due to the

211 downward $\mathbf{E} \times \mathbf{B}$ drifts outside the EPBs pushing the plasma downward. In case 4, the airglow

212 deviations of EPB in the northern hemisphere significantly become less significant compared to

213 the EPB in the southern hemisphere, displaying a hemispheric asymmetry of EPB. However, the

214 EPB is still distinct in both hemispheres, suggesting that meridional wind alone is insufficient to

215 fade the EPB out. In case 5, it shows that the EPB is almost evanescent in the northern hemisphere

216 while interacting with the MSTIDs. Simulations reveal that both MSTIDs and meridional wind

217 are likely necessary to comprehend the disappearance of EPB. 


\section{Discussion and Conclusion}

221 by transporting plasma into the depletions via $\mathbf{E} \times \mathbf{B}$ drifts. In our simulations, we found that

222 MSTIDs can suppress the upward motion of EPBs; however, MSTIDs alone are insufficient to

223 explain the disappearance of EPBs. The meridional winds should be included in the processes to

224 fully explain the disappearance of EPBs in the airglow observations. These processes involve (1)

225 the suppression of upward $\mathbf{E} \times \mathbf{B}$ drifts within the EPBs, (2) the decrease in background electron

226 density, (3) and the transport of ambient plasma by MSTIDs.

227

First, the suppression of upward $\mathbf{E} \times \mathbf{B}$ drifts occurred when EPBs interact with MSTIDs and meridional winds. Figure 4 shows the local time variations of peak vertical $\mathbf{E} \times \mathbf{B}$ drifts of the topside EPBs for cases 1-5. Cases 2 and 3 show significant fluctuations due to the interactions of upward and downward $\mathbf{E} \times \mathbf{B}$ drifts within the MSTIDs. The MSTIDs can suppress or aid the upward motion of EPBs by reducing or increasing the upward $\mathbf{E} \times \mathbf{B}$ drifts in comparison with 
234 (2009b) and Huba and Krall (2013) suggested that a uniform meridional wind perpendicular to

235 the magnetic field has a direct stabilizing effect on EPB development. The meridional wind

236 parallel to the magnetic field can also indirectly stabilize the EPBs by altering the Pedersen

237 conductivity. Figure 5 shows the distributions of Pedersen conductivity (top) and vertical $\mathbf{E} \times \mathbf{B}$

238 drift (bottom) as a function of latitude and altitude in $2^{\circ} \mathrm{E}$ for case 1 (left), case 4 (middle), and

239 case 5 (right) at 01:49 LT. In comparison with case 1, case 4 shows that the Pedersen conductivity

240 is decreased (increased) when the southward winds move the plasma to higher (lower) altitudes

241 in the northern (southern) hemisphere. The increase of electron density in lower altitude will lead

242 to the increase of field-line integrated Pedersen conductivity since the Pedersen conductivity is

243 proportional to electron and neutral densities (Kelley, 1989), which, in turn, suppress the EPB

244 growth (e.g., Huba et al. 2020). Case 5 also shows that the MSTIDs can increase (decrease) the

245 Pedersen conductivity in the bottomside ionosphere (below $300 \mathrm{~km}$ ) by transporting the plasma

246 to lower (higher) altitude via downward (upward) $\mathbf{E} \times \mathbf{B}$ drifts, which is consistent with the

247 Perkins instability.

248 Second, the meridional winds can significantly reduce the background electron density and

249 airglow intensity. The southward winds move the plasma upward (downward) in the northern 
250 (southern) hemisphere, leading to a decrease (increase) of plasma density and airglow intensity

251 below the F region. The top panel of Figure 3 shows that the EPBs in both hemispheres are

252 asymmetric. The relative airglow deviations of EPB become smaller in the northern hemisphere,

253 demonstrating that the decrease in plasma density and airglow intensity would make EPB

254 inconspicuous, providing conditions favorable for MSTIDs to fade out the EPBs.

255 Third, since the EPBs usually have large upward $\mathbf{E} \times \mathbf{B}$ drifts, MSTIDs can efficiently fade

256 the EPBs out when the upward $\mathbf{E} \times \mathbf{B}$ drifts within the EPBs and background electron density are

257 reduced. The decrease in background electron density can also lead to a decrease in upward

$258 \mathbf{E} \times \mathbf{B}$ drifts within the EPBs (e.g., Krall et al., 2010). Then, the MSTIDs move plasma downward

259 to fill the EPB depletions up via $\mathbf{E} \times \mathbf{B}$ drifts, which, in turn, lead to the enhancement of the 630-

$260 \mathrm{~nm}$ airglow intensity (bottom panel of Figure 3). Our simulated OI 630-nm results essentially

261 agree with the airglow observations reported by Otsuka et al. (2012) and Shiokawa et al. (2015).

262 It should be mentioned that Otsuka et al. (2012) showed that the EPB is nearly the fossil state

263 when encountering the MSTIDs, implying that the upward $\mathbf{E} \times \mathbf{B}$ drifts within the EPB should be

264 weak. The MSTIDs and meridional winds should be more efficiently suppressing the EPB.

265 Although the meridional winds and MSTIDs contribute to the disappearance of EPBs in the 
266 OI 630-nm airglow observations, Figure 4 shows clear depletions in Pedersen conductivity above

$267 \sim 300 \mathrm{~km}$ in the northern hemisphere (case 5), suggesting that the EPBs still persist due to

268 abundant background electron density in the F region. This can explain why the strong spread-F

269 was still present in ionograms when the EPBs were disappeared in the airglow images (Shiokawa

270 et al. 2015).

271 On the other hand, we found that MSTIDs can also lead to west-tilted EPBs. The west-tilted

272 EPBs are usually considered to be related to the latitudinal variation of eastward plasma flow due

273 to zonal wind (Kelley et al. 2003; Huba et al. 2009b). The eastward plasma flow peaks at the

274 equatorial region can lead to a reverse C- or arc-shape EPBs (Kil et al. 2009). Figure 6 shows the

275 variation of zonal $\mathbf{E} \times \mathbf{B}$ drifts as a function of longitude and altitude for cases 1 (top panel) and

2762 (bottom panel). In case 1, the primary plume tilts eastward due to the eastward $\mathbf{E} \times \mathbf{B}$ drifts

277 within the EPB. The bifurcations on the west wall of the plume can be identified with the

278 westward $\mathbf{E} \times \mathbf{B}$ drifts. In case 2, the primary plume initially grows eastward due to the eastward

$279 \mathbf{E} \times \mathbf{B}$ drifts within the EPB (Figure 6e). As the EPB grows upward and encounters the MSTIDs

280 with westward $\mathbf{E} \times \mathbf{B}$ drifts, the primary plume turns westward and stretches along with the band

281 structure of MSTIDs (Figures 6f-h). The bifurcations on the east wall of the plume are mainly 
eastward while encountering the MSTIDs with eastward $\mathbf{E} \times \mathbf{B}$ drifts. However, they are

283 suppressed by the downward $\mathbf{E} \times \mathbf{B}$ drifts of MSTIDs as shown in Figure 1. A similar

284 phenomenon was observed by Aa et al. (2019) that they found the EPBs merged and stretched

285 along with the mid-latitude TIDs.

286 In conclusion, we study the disappearance of EPBs due to the influences of MSTIDs by using

287 the SAMI3/ESF model. Simulations reveal that MSTIDs alone are insufficient to explain the

288 disappearance of EPBs. The inclusion of meridional winds is necessary to comprehend the

289 underlying mechanism. Both MSTIDs and meridional winds can suppress the upward $\mathbf{E} \times \mathbf{B}$ drifts

290 within the EPBs and reduce the background electron density, providing conditions favorable for

291 the MSTIDs to fill the plasma depletion up through $\mathbf{E} \times \mathbf{B}$ drifts. Besides, the vertical and zonal

$292 \mathbf{E} \times \mathbf{B}$ drifts within the MSTIDs can affect the morphology of EPBs in the onset phase, making

293 EPBs stretch along with the band structure of MSTIDs and display a reverse C-shape.

294

295 Declarations

296 Ethics approval and consent to participate

297 Not applicable

298 Consent for publication 
299 Not applicable

$300 \quad$ List of abbreviations

301 MSTIDs: Medium-Scale Traveling Ionospheric Disturbances

302 EPBs: Equatorial Plasma Bubbles

303 PRE: Pre-Reversal Enhancement

304 EsL: Sporadic-E Layer

305 NRL: Naval Research Laboratory

306 SAMI3/ESF: Sami3 is Also a Model of the Ionosphere/Equatorial Spread F

307 SAMI2: Sami2 is Another Model of the Ionosphere

308 LT: Local Time

$309 \quad$ Availability of data and materials

310 The datasets used during this study were constructed from the SAMI3/ESF model and

311 are available from the corresponding author on reasonable request.

\section{Competing interests}

313 The authors declare that they have no competing interests.

\section{$314 \quad$ Funding}


315 This study is supported by the Taiwan Ministry of Science and Technology under MOST-108-

316 2638-M-006-001-MY2. MYC is supported by National Science Foundation grant AGS-

317 1522830. JDH was supported by NASA grant NNH17ZDA001N and National Science

318 Foundation grant AGS-1931415.

\section{Authors' contributions}

320 MYC conducted the model simulations and drafted the manuscript. CHL provided

321 expertise in interpreting the modeling results and revised the manuscript. JDH developed

322 the model, provided expertise in interpreting the modeling results, and revised the

323 manuscript. All authors read and approved the final manuscript.

\section{Acknowledgements}

325 The authors acknowledge Naval Research Laboratory and Dr. J. D. Huba for providing the

326 SAMI3/ESF model. The authors thank two anonymous reviewer and editor for their helpful

327 comments and suggestions on improving this manuscript.

328 Authors' information

329 COSMIC Program Office, University Corporation for Atmospheric Research, Boulder, CO,

$330 \quad$ USA 
333 Charles C. H. Lin

334 Syntek Technologies, Fairfax, VA, USA

335 Joseph D. Huba

\section{References}

337 Aa, E., Zou, S., Ridley, A. J., Zhang, S.-R., Coster, A. J., Erickson, P. J., et al. (2019).

338 Merging of storm time midlatitude traveling ionospheric disturbances and equatorial plasma

339 bubbles. Space Weather, 17. https://doi.org/10.1029/2018SW002101.

340 Abdu, M. A., E. Alam Kherani, I. S. Batista, E. R. de Paula, D. C. Fritts, and J. H. A. Sobral

341 (2009), Gravity wave initiation of equatorial spread F/plasma bubble irregularities based on observational data from the SpreadFEx campaign, Ann. Geophys., 27, 2607, doi:10.5194/angeo-27-2607-2009.

344 Behnke, R. (1979), F layer height bands in the nocturnal ionosphere over Arecibo, J. Geophys. Res., 84, 974-978, doi:10.1029/JA084iA03p00974. 
traveling ionospheric disturbances triggered by Super Typhoon Nepartak (2016), Geophys.

Chou, M.-Y., Lin, C. C. H., Huba, J. D., Lien, C.-P., Chen, C.-H., Yue, J., et al. (2018). Numerical

353 Cosgrove, R. B., and R. T. Tsunoda (2004), Instability of the E-F coupled nighttime midlatitude ionosphere, J. Geophys.Res., 109, A04305, doi:10.1029/2003JA010243.

355 Drob, D. P., et al. (2015), An update to the horizontal wind model (HWM): The quiet time thermosphere, Earth Space Sci., 2, 301-319, doi:10.1002/2014EA000089.

357 Dungey, J. W. (1956), Convective diffusion in the equatorial F-region, J. Atmos. Terr. Phys., 9(56), 304-310.

Duly, T. M., J. D. Huba, and J. J. Makela (2014), Self-consistent generation of MSTIDs within the SAMI3 numerical model, J. Geophys. Res. Space Physics, 119, 6745-6757, doi:10.1002/2014JA020146. 
364 Huba, J. D., G. Joyce, and J. Krall (2008), Three-dimensional equatorial spread F modeling, Geophys. Res. Lett., 35, L10102, doi:10.1029/2008GL033509.

Huba, J. D., G. Joyce, J. Krall, and J. Fedder (2009a), Ion and electron temperature evolution during equatorial spread F, Geophys. Res. Lett., 36, L15102, doi:10.1029/2009GL038872. equatorial spread F modeling: Zonal neutral wind effects, Geophys. Res. Lett., 36, L19106, doi:10.1029/2009GL040284.

Huba, J. D., and G. Joyce (2010), Global modeling of equatorial plasma bubbles, Geophys. Res. Lett., 37, L17104, doi:10.1029/2010GL044281.

Huba, J. D., and Krall, J. ( 2013), Impact of meridional winds on equatorial spread F: Revisited, Geophys. Res. Lett., 40, 1268-1272, doi:10.1002/grl.50292.

Huba, J. D., Krall, J., \& Drob, D. (2020). Modeling the impact of metallic ion layers on equatorial spread F with SAMI3/ESF. Geophysical Research Letters, 47, e2020GL087224. 
Kelley, M. C., Makela, J. J., Paxton, L. J., Kamalabadi, F., Comberiate, J. M., and Kil, H. (2003), bubbles, Geophys. Res. Lett., 30, 1766, doi:10.1029/2003GL017301, 14.

384 Kelley, M. C., The Earth's Ionosphere: Plasma Physics and Electrodynamics, Academic, San Diego, Calif., 1989.

Kelley, M. C., J. J. Makela, A. Saito, N. Aponte, M. Sulzer, and S. A. González (2000), On the electrical structure of airglow depletion/height layer bands over Arecibo, Geophys. Res. Lett., 27(18), 2837-2840.

Kil, H., Heelis, R. A., Paxton, L. J., and Oh, S.-J. ( 2009), Formation of a plasma depletion shell in the equatorial ionosphere, J. Geophys. Res., 114, A11302, doi:10.1029/2009JA014369. GPS total electron content variations caused by medium-scale traveling ionospheric disturbances, J. Geophys. Res., 111, A04306, doi:10.1029/2005JA011418.

394 Krall, J., Huba, J. D., and Martinis, C. R. (2009a), Three-dimensional modeling of equatorial 
spread F airglow enhancements, Geophys. Res. Lett., 36, L10103, doi:10.1029/2009GL038441.

Krall, J., J. D. Huba, G. Joyce, and S. T. Zalesak (2009b), Three - dimensional simulation of equatorial spread F with meridional wind effects, Ann. Geophys., 27, 1821.

Krall, J., J. D. Huba, S. L. Ossakow, and G. Joyce (2010), Why do equatorial ionospheric bubbles stop rising? Geophys. Res. Lett., 37, L09105, doi:10.1029/2010GL043128. Modeling of equatorial plasma bubbles triggered by non- equatorial traveling ionospheric disturbances, Geophys. Res. Lett., 38, L08103, doi:10.1029/2011GL046890.

404 Krall, J., J. D. Huba, G. Joyce, and M. Hei (2013), Simulation of the seeding of equatorial spread F by circular gravity waves, Geophys. Res. Lett., 40, 1-5, doi: 10.1029/ 2012GL054022. variations induced by pre-earthquake rock currents and associated surface charges, J. Geophys. Res., 116, A10317, doi:10.1029/2011JA016628. 
412 Martinis, C., Baumgardner, J., Mendillo, M., Wroten, J., Coster, A., and Paxton, L. ( 2015), The

413 night when the auroral and equatorial ionospheres converged, J. Geophys. Res. Space Physics,

$414 \quad 120,8085-8095$, doi:10.1002/2015JA021555.

415 Miller, C. A., Swartz, W. E., Kelley, M. C., Mendillo, M., Nottingham, D., Scali, J., and Reinisch,

416 B. (1997), Electrodynamics of midlatitude spread F: 1. Observations of unstable, gravity

417 wave - induced ionospheric electric fields at tropical latitudes, J. Geophys. Res., 102( A6),

$418 \quad$ 11521-11532, doi:10.1029/96JA03839.

419 Nishioka, M., A. Saito, and T. Tsugawa (2008), Occurrence characteristics of plasma bubble

420 derived from global ground-based GPS receiver networks, J. Geophys. Res., 113, A05301,

421 doi:10.1029/2007JA012605.

422 Otsuka, Y., K. Shiokawa, T. Ogawa, and P. Wilkinson (2002), Geomagnetic conjugate

423 observations of equatorial airglow depletions, Geophys. Res. Lett., 29(15), doi:10.1029/2002GL015347. 
Otsuka, Y., K. Shiokawa, and T. Ogawa (2012), Disappearance of equatorial plasma bubble after interaction with mid-latitude medium-scale traveling ionospheric disturbance, Geophys. Res. Lett., 39, L14105, doi:10.1029/2012GL052286.

Ogawa, T., E. Sagawa, Y. Otsuka, K. Shiokawa, T. J. Immel, S. B. Mende, and P. Wilkinson (2005), Simultaneous ground- and satellite-based airglow observations of geomagnetic conjugate plasma bubbles in the equatorial anomaly, Earth Planets Space, 57, 385-392.

434 Picone, J. M., A. E. Hedin, D. P. Drob, and A. C. Aikin (2002), NRLMSISE - 00 empirical model of the atmosphere: Statistical comparisons and scientific issues, J. Geophys. Res., 107(A12), 1468, doi:10.1029/2002JA009430. Iwagami, N.: Electric field measurements of DC and long wavelength structures associated with sporadic-E layers and QP radar echoes, Ann. Geophys., 23, 2319-2334, https://doi.org/10.5194/angeo-23-2319-2005, 2005.

441 Perkins, F. (1973), Spread F and ionospheric currents, J. Geophys. Res., 78, 218, 
443 Rama Rao, P. V. S., P. T. Jayachandran, and P. S. Ram (1997), Ionospheric irregularities: The role

444 of the equatorial ionization anomaly, Radio Sci., 32(4), 1551-1557, doi:10.1029/97RS00665.

445 Rajesh, P. K., J. Y. Liu, C. H. Lin, A. B. Chen, R. R. Hsu, C. H. Chen, and J. D. Huba (2016),

446 Space-based imaging of nighttime medium-scale traveling ionospheric disturbances using

447 FORMOSAT-2/ISUAL 630.0 nm airglow observations, J. Geophys. Res. Space Physics, 121,

$448 \quad$ 4769-4781, doi:10.1002/2015JA022334.

449 Rajesh, P. K., C. H. Lin, C. H. Chen, J. T. Lin, T. Matsuo, M. Y. Chou, W. H. Chen, M. T. Chang,

450 and C. F. You (2017), Equatorial plasma bubble generation/inhibition during 2015 St.

451 Patrick's Day storm, Space Weather, 15, 1141-1150, doi:10.1002/2017SW001641.

452 Saito, A., T. Iyemori, M. Sugiura, N. C. Maynard, T. L. Aggson, L. H. Brace, M. Takeda, and M.

453 Yamamoto (1995), Conjugate occurrence of the electric field fluctuations in the nighttime

454 midlatitude ionosphere, J. Geophys. Res., 100(A11), 21,439-21,451, doi:10.1029/95JA01505.

456

Saito, A., T. Iyemori, L. G. Blomberg, M. Yamamoto, and M. Takeda (1998), Conjugate 
459 Shiokawa, K., C. Ihara, Y. Otsuka, and T. Ogawa (2003a), Statistical study of nighttime mediumscale traveling ionospheric disturbances using midlatitude airglow images, J. Geophys. Res., 108(A1), 1052, doi:10.1029/2002JA009491.

Shiokawa, K., Otsuka, Y., Ihara, C., Ogawa, T., \& Rich, F. J. (2003b). Ground and satellite observations of nighttime medium-scale traveling ionospheric disturbance at midlatitude. Journal of Geophysical Research, 108(A4), 1145. https://doi.org/10.1029/2002JA009639.

Shiokawa, K., Y. Otsuka, K. J. W. Lynn, P. Wilkinson, and T. Tsugawa (2015), Airglow-imaging observation of plasma bubble disappearance at geomagnetically conjugate points, Earth Planets Space, 67, 43, doi:10.1186/s40623-015-0202-6.

Sobral, J. H. A., Takahashi, H., Abdu, M. A., Muralikrishna, P., Sahai, Y., Zamlutti, C. J., de Paula, E. R., and Batista, P. P. ( 1993), Determination of the quenching rate of the O( $\left.{ }^{1} \mathrm{D}\right)$ by $\mathrm{O}\left({ }^{3} \mathrm{P}\right)$ from rocket-borne optical $(630 \mathrm{~nm})$ and electron density data, J. Geophys. Res., 98( A5), 7791- 7798, doi:10.1029/92JA01839. 

disturbances detected with dense and wide TEC maps over North America, Geophys. Res. Lett., 34, L22101, doi:10.1029/2007GL031663. missing link found, J. Geophys. Res., 109, A12303, doi:10.1029/2004JA010597. Sci. 2, 9 (2015). https://doi.org/10.1186/s40645-015-0038-5 
491 Yokoyama, T., Shinagawa, H., and Jin, H. (2015), Nonlinear growth, bifurcation and pinching of equatorial plasma bubble simulated by three - dimensional high -

493 resolution bubble model, J. Geophys. Res. Space Physics, 119, pages 10,474-

494 10,482. doi:10.1002/2014JA020708.

\section{$495 \quad$ Figure legends}

496 Figure 1. The time sequence of electron density contours as a function of longitude and altitude

497 at 22:47 LT, 23:48 LT, 00:48 LT, and 01:49 LT for case 1 (left column), case 2 (middle column),

498 and case 3 (right column). The black and white contour lines indicate the downward and upward

$499 \mathbf{E} \times \mathbf{B}$ drifts, respectively. The yellow and red stars indicate the positions of peak upward $\mathbf{E} \times \mathbf{B}$

500 drifts of the EPB and topside EPB $(>900 \mathrm{~km})$. Case 1 simulates the EPB without the MSTIDs.

501 Case 2 and case 3 simulate the interaction between the EPB and MSTIDs at 22:30 LT and 23:30

502 LT, respectively.

503

504 Figure 2 . The time sequence of airglow intensity perturbation contours at $250 \mathrm{~km}$ altitude as a

505 function of longitude and latitude at 22:47 LT, 23:48 LT, 00:48 LT, and 01:49 LT for case 1 (first

506 column), case 2 (second column), and case 3 (third column). 
508 Figure 3 . The time sequence of airglow intensity perturbation contours at $250 \mathrm{~km}$ altitude as a

509 function of longitude and latitude at 22:48 LT, 23:48 LT, 00:48 LT, and 01:48 LT for case 4 (top

510 panel) and case 5 (bottom panel).

511

512 Figure 4. The peak vertical $\mathbf{E} \times \mathbf{B}$ drift variations of the topside EPBs ( $>900 \mathrm{~km})$ as a function

513 of time for cases 1-5.

514

515 Figure 5. The Pedersen conductivity contours as a function of latitude and altitude in $\sim 2^{\circ} \mathrm{E}$ at

516 01:49 LT for case 1 (left), case 4 (middle), and case 5 (right). The white (top panel) and black

517 lines (bottom panel) indicate the geomagnetic field line.

518

519 Figure 6. The time sequence of zonal $\mathbf{E} \times \mathbf{B}$ drift contours as a function of longitude and altitude

520 for case 2 at 22:47 LT, 23:48 LT, 00:48 LT, and 01:49 LT for cases 1 (top panel) and 2 (bottom

521 panel). The positive and negative values indicate eastward and westward $\mathbf{E} \times \mathbf{B}$ drifts,

522 respectively. 


\section{Additional files}

525 - File name: Additional file 1, Additional file 2, Additional file 3, Additional file

5264 , Additional file 5, Additional file 6, Additional file 7, Additional file 8

527 - File format: avi

528 - Title of data: Electron density variations as a function of longitude and altitude for

529 cases 1-3 (Additional files 1-3). Airglow intensity perturbation variations at an altitude

530 of $250 \mathrm{~km}$ as a function of longitude and latitude for cases 1-5 (additional files 4-8).

531 - Description of data: Additional files 1-3 are movies for Figure 1. Additional

532 files 4-6 are movies for Figure 2. Additional files 7-8 are movies for Figure 3.

533 


\section{Figures}
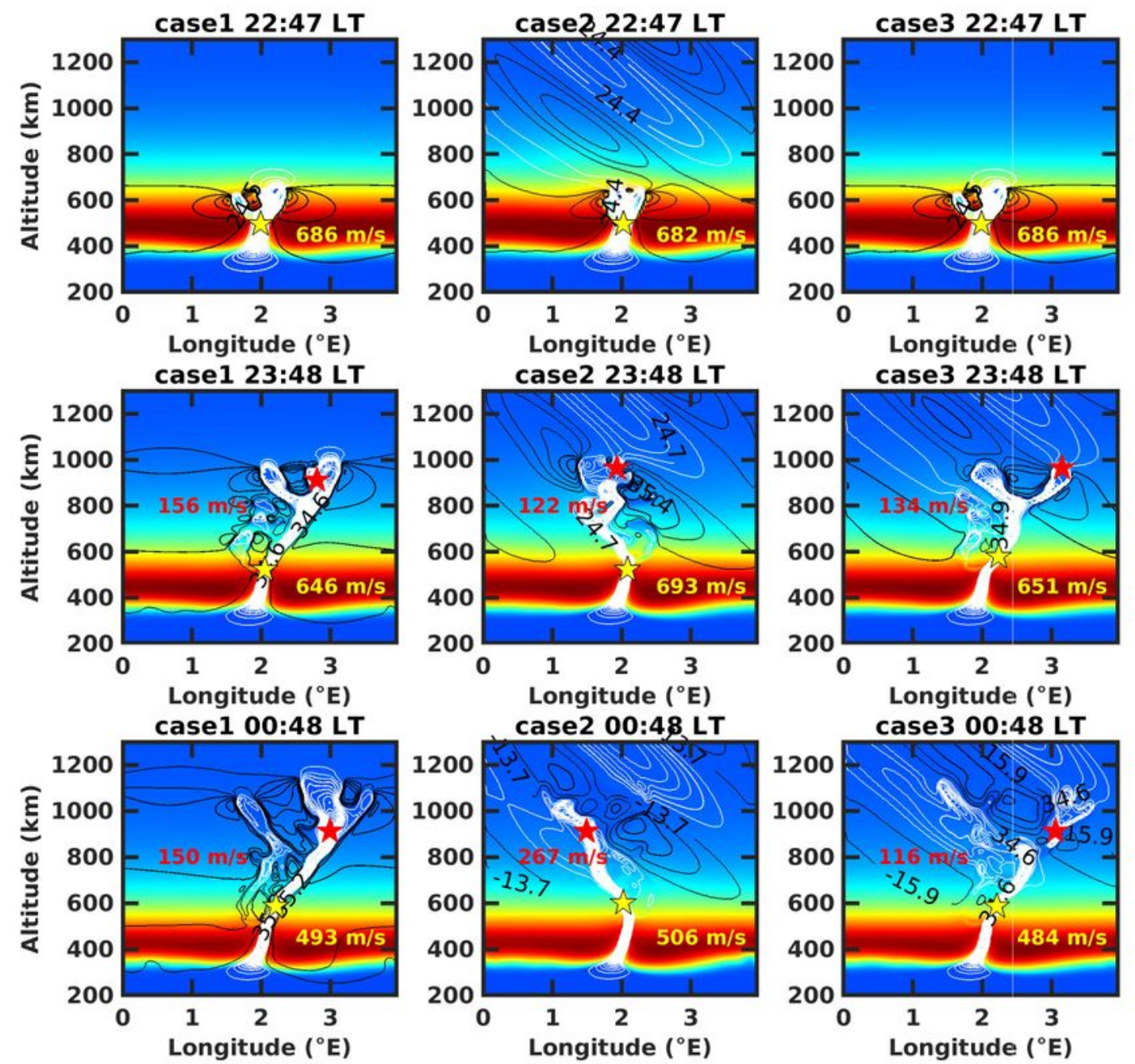

Longitude $\left({ }^{\circ} \mathrm{E}\right)$
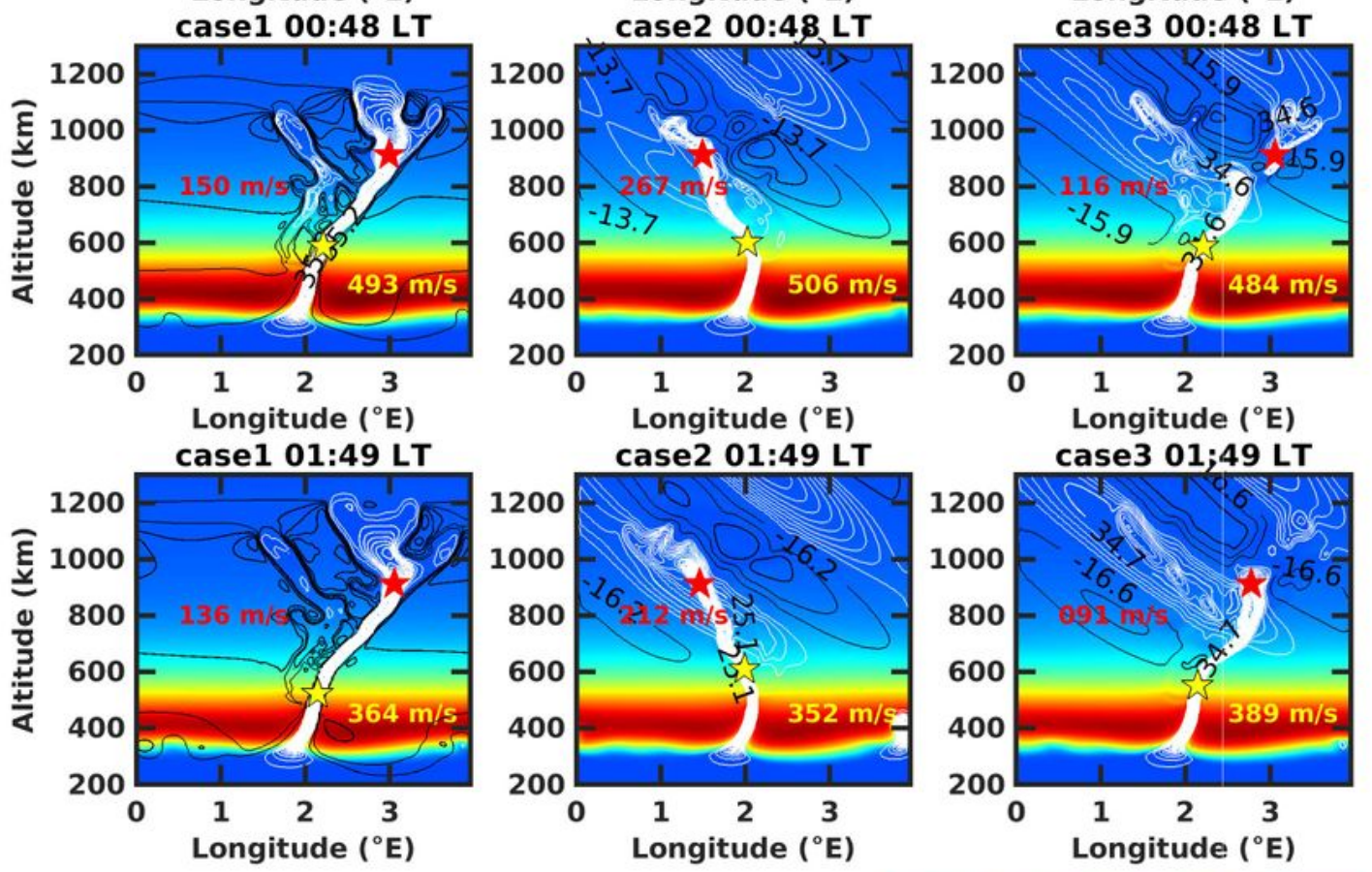

Longitude $\left({ }^{\circ} \mathrm{E}\right)$
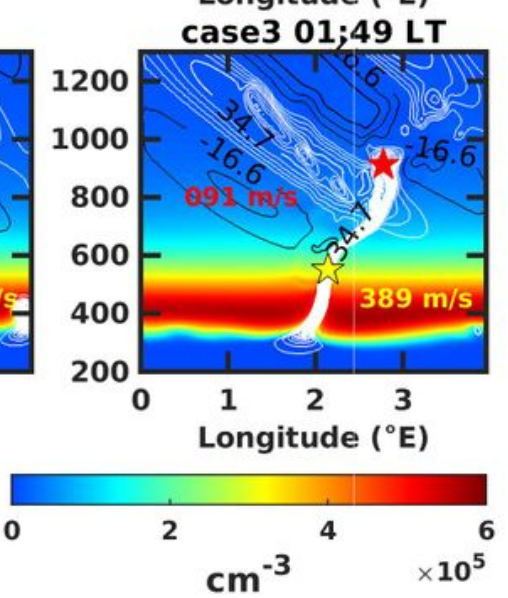

Figure 1

The time sequence of electron density contours as a function of longitude and altitude at 22:47 LT, 23:48 LT, 00:48 LT, and 01:49 LT for case 1 (left column), case 2 (middle column), and case 3 (right column). The black and white contour lines indicate the downward and upward E $\times \mathrm{B}$ drifts, respectively. The 
yellow and red stars indicate the positions of peak upward E $\times B$ drifts of the EPB and topside EPB (>900 $\mathrm{km})$. Case 1 simulates the EPB without the MSTIDs. Case 2 and case 3 simulate the interaction between the EPB and MSTIDs at 22:30 LT and 23:30 LT, respectively.
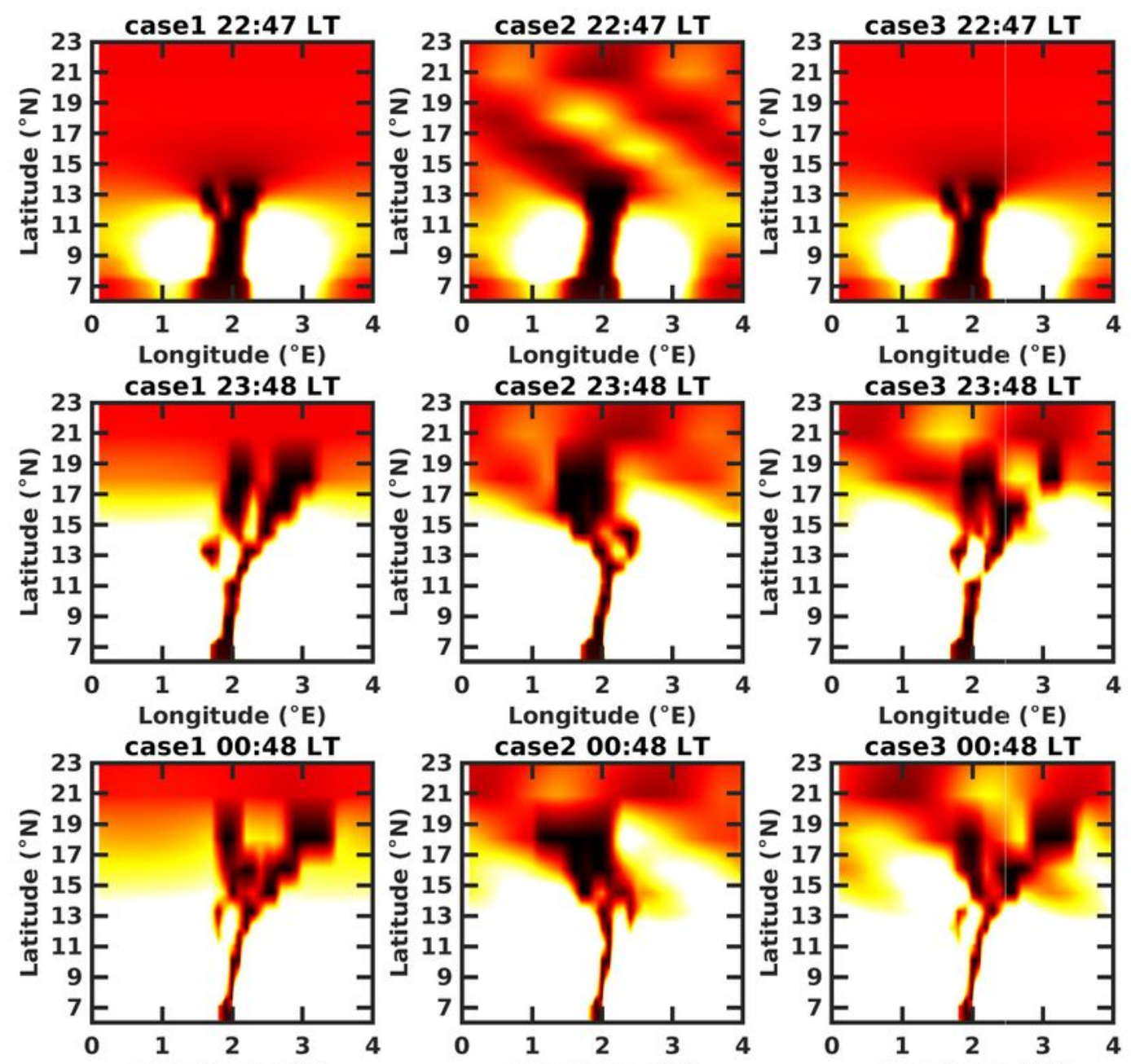

Longitude ( $\left.{ }^{\circ} \mathrm{E}\right)$
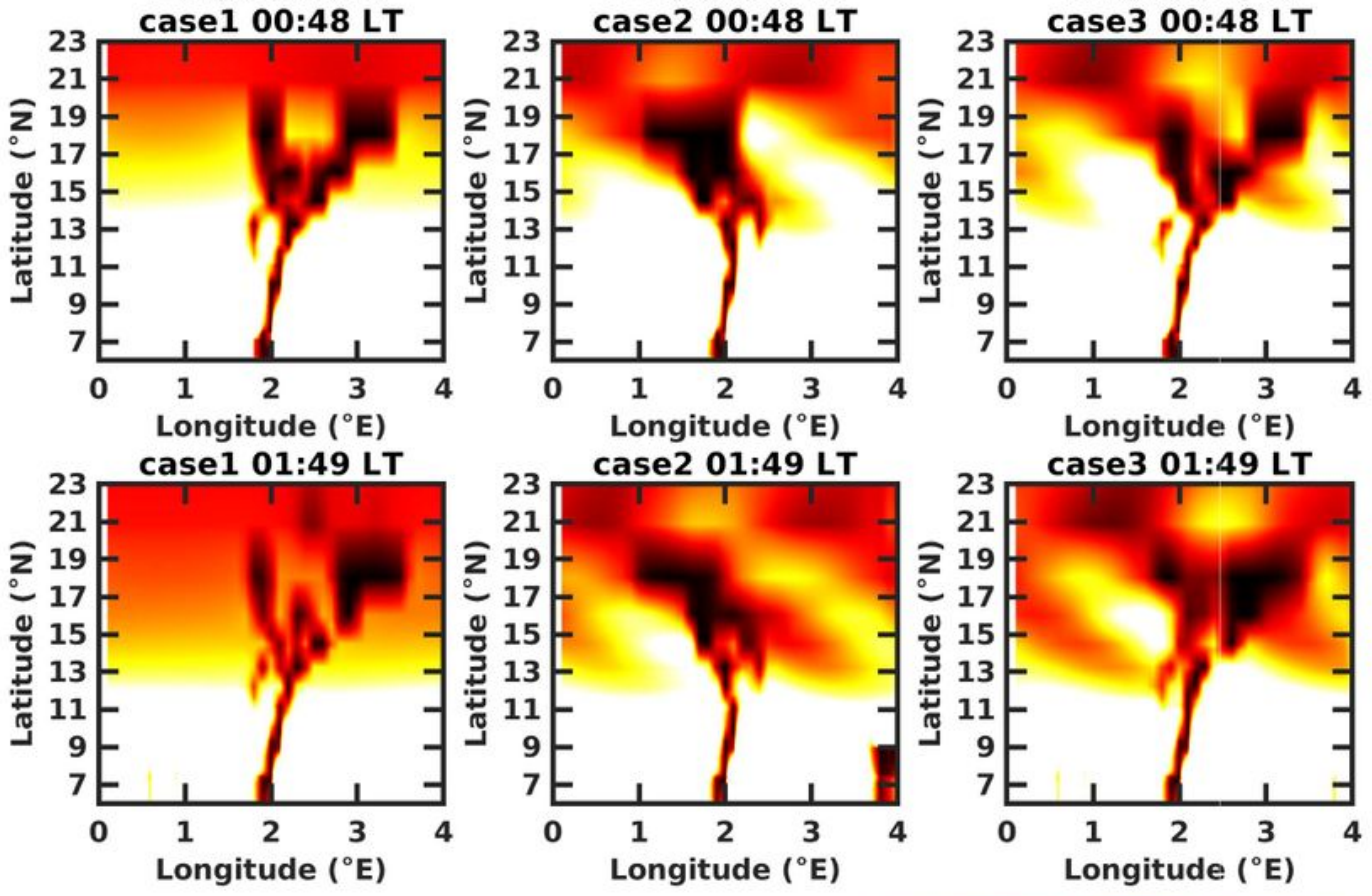

Longitude $\left({ }^{\circ} \mathrm{E}\right)$

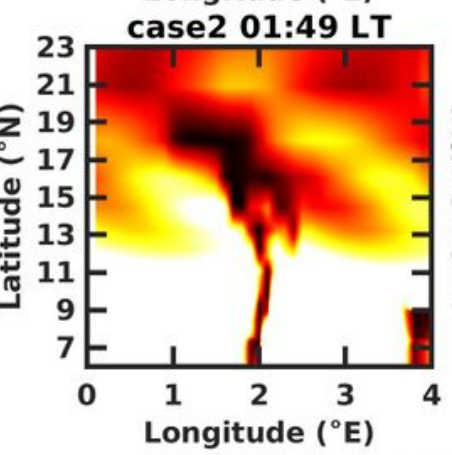

Longitude $\left({ }^{\circ} \mathrm{E}\right)$

case 3 01:49 LT
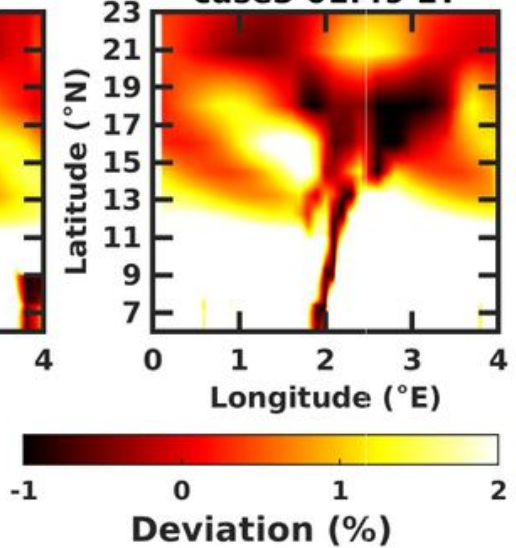

Figure 2

The time sequence of airglow intensity perturbation contours at $250 \mathrm{~km}$ altitude as a function of longitude and latitude at 22:47 LT, 23:48 LT, 00:48 LT, and 01:49 LT for case 1 (first column), case 2 

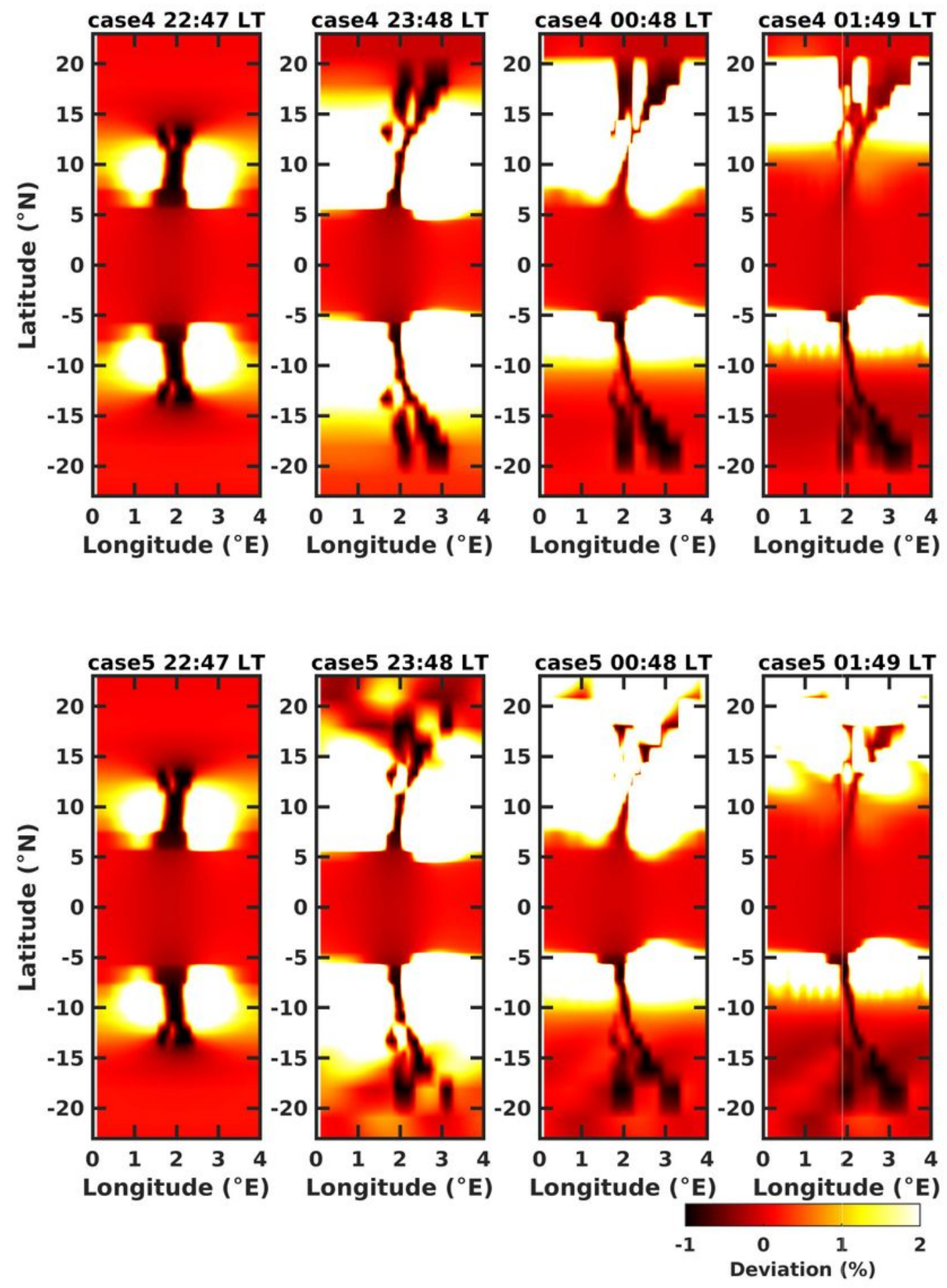

Figure 3

The time sequence of airglow intensity perturbation contours at $250 \mathrm{~km}$ altitude as a function of longitude and latitude at 22:48 LT, 23:48 LT, 00:48 LT, and 01:48 LT for case 4 (top panel) and case 5 (bottom panel). 


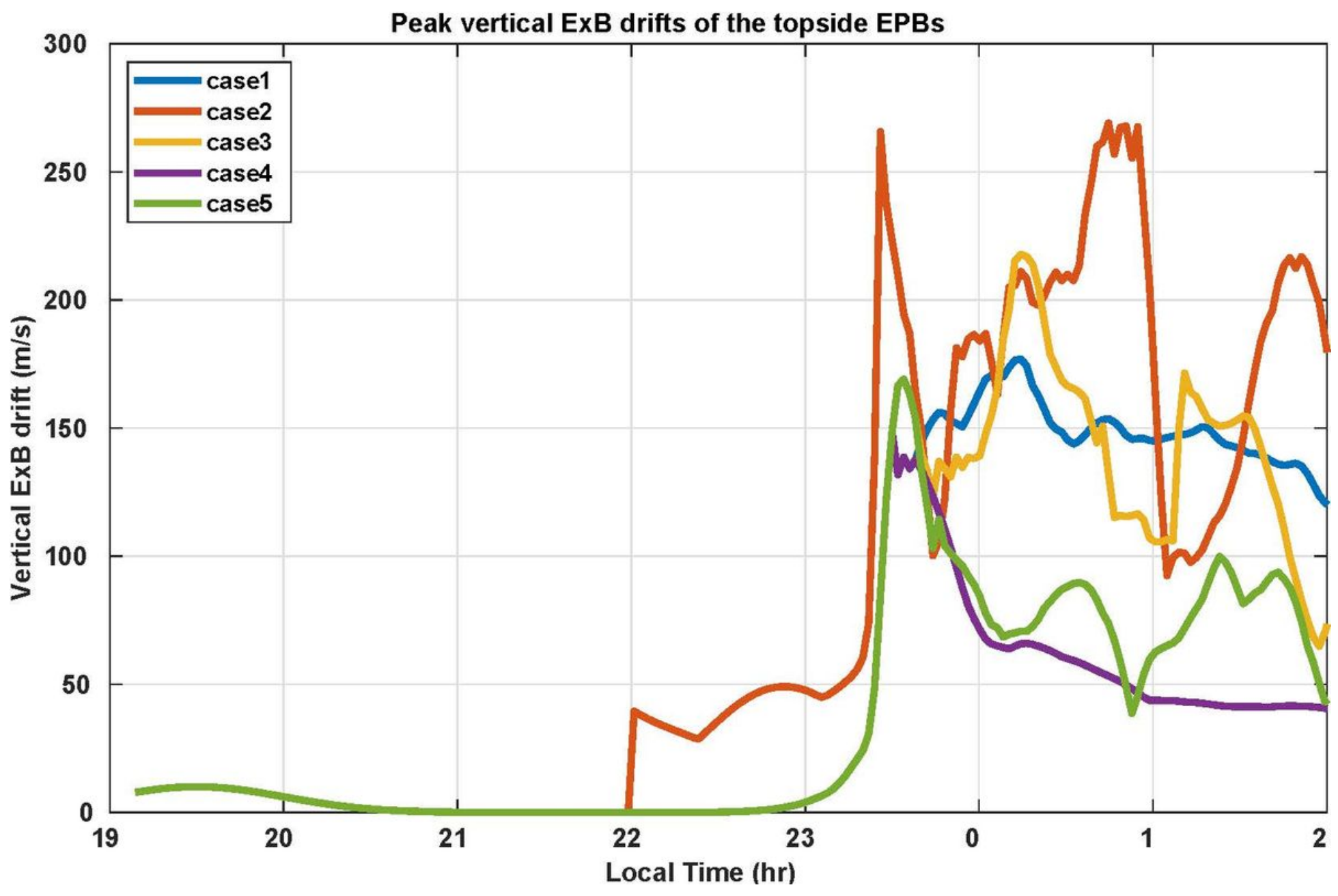

Figure 4

The peak vertical E $\times$ B drift variations of the topside EPBs $(>900 \mathrm{~km})$ as a function of time for cases 1-5. 

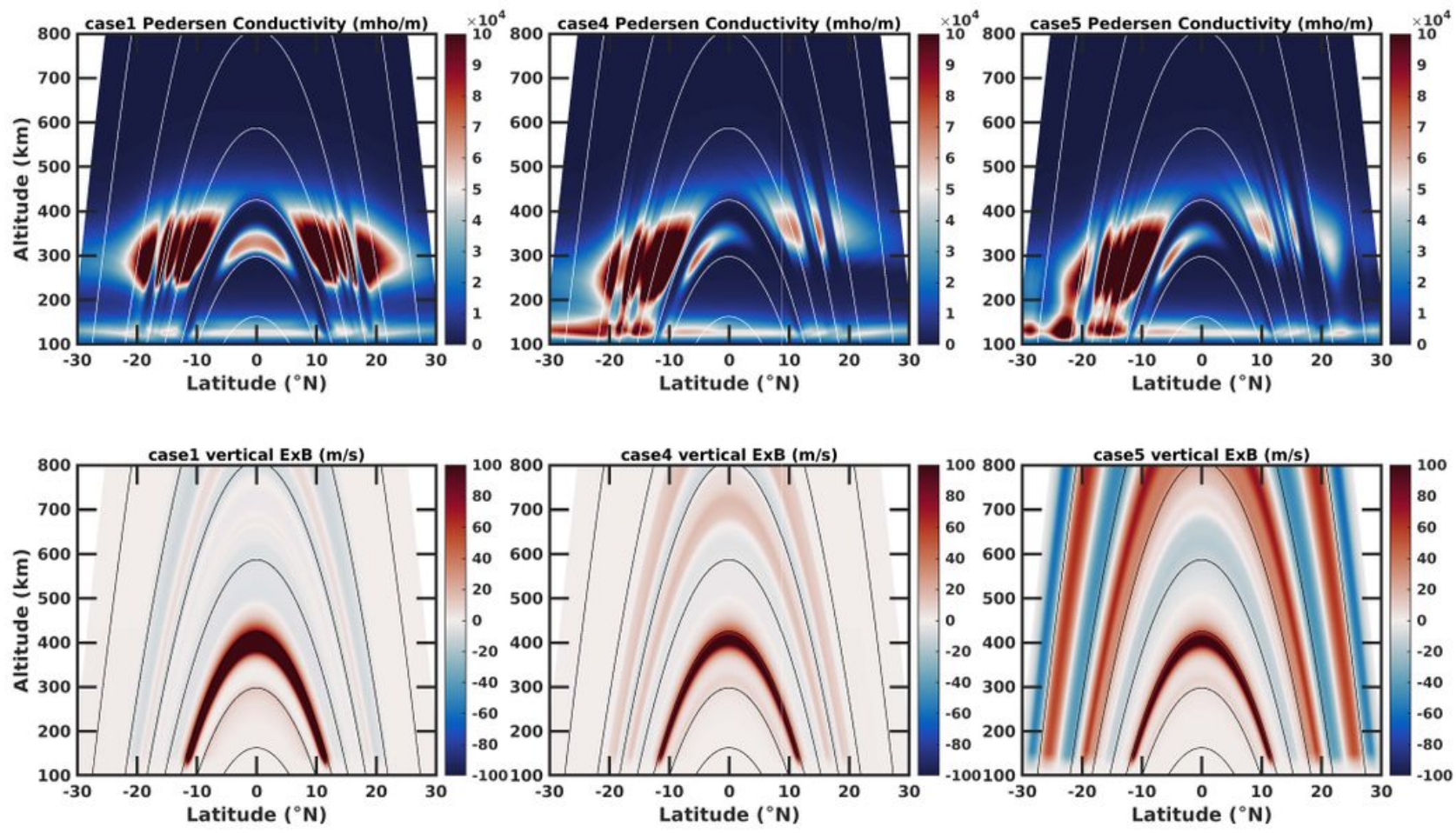

\section{Figure 5}

The Pedersen conductivity contours as a function of latitude and altitude in $\sim 2^{\circ} \mathrm{E}$ at 01:49 LT for case 1 (left), case 4 (middle), and case 5 (right). The white (top panel) and black lines (bottom panel) indicate the geomagnetic field line. 

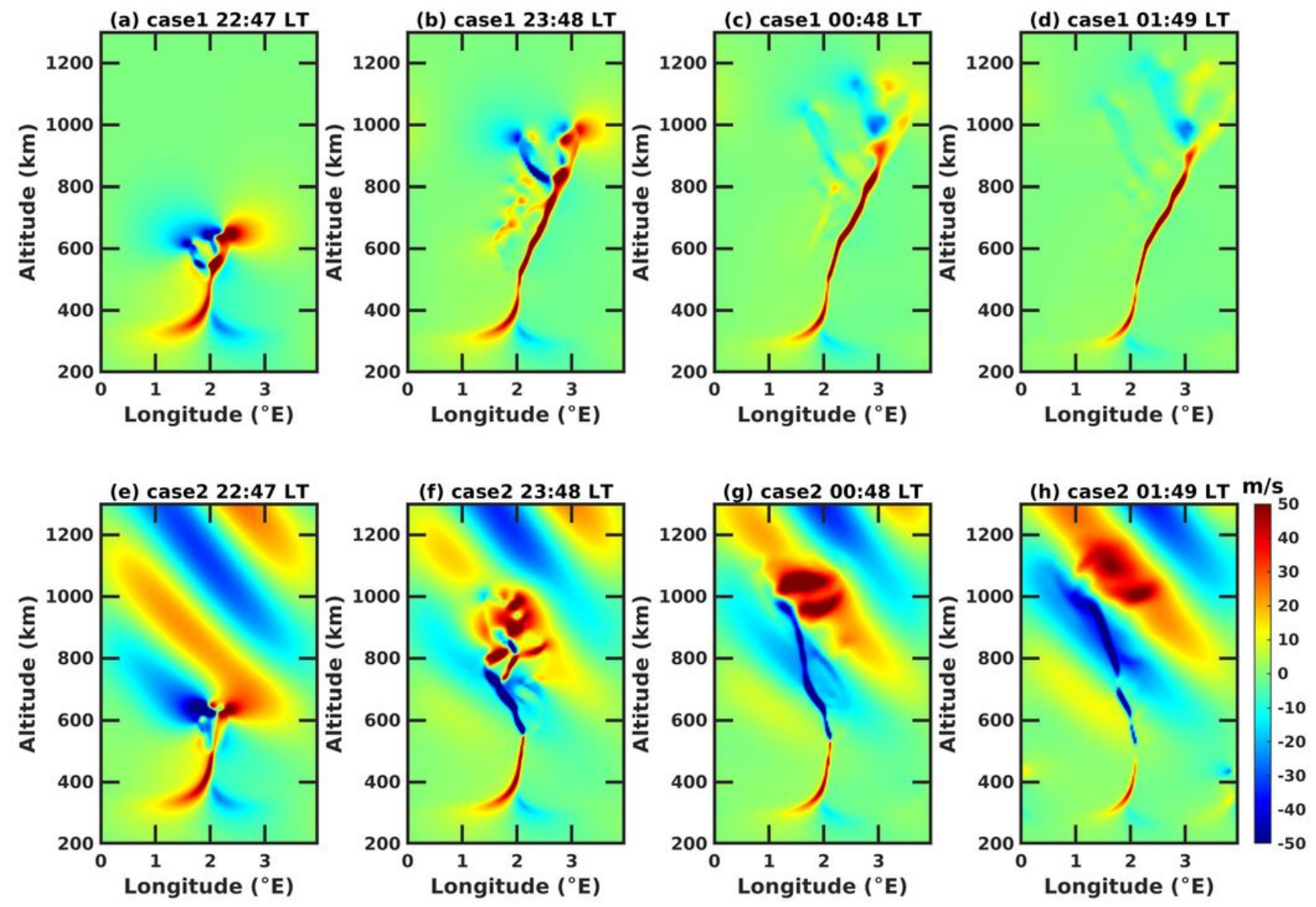

\section{Figure 6}

The time sequence of zonal $\mathrm{E} \times \mathrm{B}$ drift contours as a function of longitude and altitude for case 2 at 22:47 LT, 23:48 LT, 00:48 LT, and 01:49 LT for cases 1 (top panel) and 2 (bottom panel). The positive and negative values indicate eastward and westward $\mathrm{E} \times \mathrm{B}$ drifts, respectively.

\section{Supplementary Files}

This is a list of supplementary files associated with this preprint. Click to download.

- Figure1abstract3.png

- case1airglow.avi

- case1ne.avi

- case2ne.avi

- case2airglow.avi

- case3airglow.avi

- case3ne.avi

- case4airglow.avi 
- case5airglow.avi 